\title{
Selenium effects on antioxidant and inflammatory indices in renal ischemia-reperfusion injury in rats
}

\author{
Hassan Ahmadvand ${ }^{1,2}{ }^{\circledR}$, Esmaeel Babaeenezhad ${ }^{3,4^{*}}{ }^{\circledR}$, Hashem Nayeri $^{5}$, Zahra Zarei Nezhad ${ }^{5}$ \\ ${ }^{1}$ Razi Herbal Medicine Research Center, Faculty of Medicine, Lorestan University of Medical Sciences, Khorramabad, Iran \\ ${ }^{2}$ Department of Biochemistry, Faculty of Medicine, Lorestan University of Medical Sciences, Khorramabad, Iran \\ ${ }^{3}$ Student Research Committee, Lorestan University of Medical Sciences, Khorramabad, Iran \\ ${ }^{4}$ Student of Veterinary Medicine, Faculty of Veterinary Medicine, Lorestan University, Khorramabad, Iran \\ ${ }^{5}$ Department of Biochemistry, Falavarjan Branch, Islamic Azad University, Isfahan, Iran
}

\section{A R T I C L E IN F O}

Article Type:

Original

\section{Article History:}

Received: 10 February 2018

Accepted: 9 June 2018

Published online: 6 July 2018

\section{Keywords:}

Selenium

Renal ischemia-reperfusion

Oxidative stress

Inflammation

\begin{abstract}
A B S T R A C T
Introduction: Selenium (Se) is an antioxidant and reactive oxygen species (ROS) scavenger. Objectives: This study was conducted to evaluate the effects of Se on renal functional parameters, oxidative stress biomarkers, myeloperoxidase (MPO) activity, and the nitric oxide (NO) level in renal ischemia-reperfusion (IR) injury in rats.

Materials and Methods: Twenty-four male Wistar rats (180-200 g) were selected and subsequently divided into three groups $(\mathrm{n}=8)$; group 1 as the control group, group 2 as the untreated group (IR without treatment) and group 3 as the IR group (treated with Se $(1 \mathrm{mg} / \mathrm{kg} / \mathrm{d}$, intraperitoneally). The period of Se administration was 2 weeks before the inducing renal IR. To cause renal IR, renal pedicles were occluded by safe clamps for 45 minutes. Then, the clamps were removed and 24 hours was considered as reperfusion. After the study, blood sampling from the hearts and the removal of the left kidney was conducted immediately for biochemical measurements.

Results: Renal IR significantly increased serum levels of urea, creatinine (Cr), serum and renal malondialdehyde (MDA) levels, serum NO level, and MPO activity. It significantly decreased serum and renal glutathione (GSH) levels, serum paraoxonase 1 activity, serum and renal activities of catalase (CAT), and glutathione peroxidase (GPx). Se could reverse these findings, but the increase of paraoxonase 1 activity and the decrease of MPO activity in IR animals were not significant. Conclusion: It seems that Se has protective effects on inflammatory indices. It can ameliorate renal IR complications which are associated with oxidative stress and inflammation.
\end{abstract}

Implication for health policy/practice/research/medical education:

Our study indicated that Se could ameliorate $\mathrm{Cr}$ and urea, LPO, the activities of antioxidant enzymes, the levels of GSH, NO, and MPO activity in IR treated group. All of the authors wish, the results of this study help to improve complications related to the renal ischemia reperfusion in the patients.

Please cite this paper as: Ahmadvand H, Babaeenezhad E, Nayeri H, Zarei Nezhad Z. Selenium effects on antioxidant and inflammatory indices in renal ischemia-reperfusion injury in rats. J Renal Inj Prev. 2019;8(2):71-77. DOI: 10.15171/jrip.2019.14.

\section{Introduction}

Ischemic condition arises from declined blood supply and can lead to diminished oxygen and nutrient delivery to cells. This phenomenon causes cell damage by producing toxic metabolites and reducing cellular energy. Other circumstances, such as free radical generation especially reactive oxygen species (ROS) and inflammation that can play a role in cell damage, are initiated by the reperfusion of blood flow to the ischemic organ. Various clinical situations are related to ischemia-reperfusion injury (IRI) such as peripheral vascular disease and stroke (1). Renal IRI (RIRI) is a common event which occurs during a series of conditions such as nephrectomy, hypovolemic shock and repair of suprarenal aneurism. It has been proven that RIRI is the major reason for acute kidney injury (AKI) and it is associated with the increase of morbidity and mortality. Different hypothesis proposed some factors which are involved in the pathogenesis of RIRI including ROS generation, inflammatory cytokines, leukocytes activation and chemokines (2). Nonetheless, ROS production has the most critical role in the occurrence of RIRI among these mechanisms. ROS has a crucial role in cell damage caused by IR (3). Therefore, the administration of antioxidants as a useful therapeutic solution can play a vital role in the treatment of RIRI. One of these antioxidants is selenium (Se). Se is recognized 
as a requirement trace element which displays various biological roles in the body including the contribution as the cofactor of selenoproteins, ROS scavenging, thyroid hormone production and anti-inflammatory effects. Se is plentifully found in seafood, meat products, and cereals. Several studies have been showed that the lack of Se in the body can participate in the pathogenesis of some disorders for example cancer, and metabolic diseases (4, 5). Recently, the role of the kidney has been demonstrated in Se metabolism because of the synthesis of various selenoproteins such as glutathione peroxidase (GPx) in the kidney. There is a significant correlation between the decreased level of Se and defects in selenoproteins. Today, it is proposed that chronic diseases can be reduced by using Se supplements (6).

\section{Objectives}

The objectives of this study were to evaluate of Se effects on antioxidant and inflammatory indices in RIRI in rats.

\section{Materials and Methods}

Chemicals

The utilized chemicals are listed as follow: Se (Sigma Aldrich Company, USA), Tris-EDTA (Merck Company, Germany), DNTB (Sigma Aldrich Company, USA), K2HPO4 (Sigma Aldrich Company, USA), H2O2 (Sigma Aldrich Company, USA), Tris-Hcl (Merck Company, Germany), NaN3 (Sigma Aldrich Company, USA), GSH (Sigma Aldrich Company, USA).

\section{Animals and study design}

24 adult male Wistar rats weighing 180-200 g were acquired from the Razi herbal medicine research center of the Lorestan University of Medical Sciences. Then, they were accustomed for one week in laboratory condition. Organized access to food and water were made for the rats. Appropriate temperature $\left(22^{\circ} \mathrm{C}\right)$ and a 12-hour light and 12-hour dark cycle condition were prepared for the rats. Finally, the rats were categorized haphazardly into three groups $(n=8)$ as follows:

Group1; Control group (without IR).

Group2; Untreated group (IR without treatment).

Group3; Treated group (treated with Se $1 \mathrm{mg} / \mathrm{kg}$ ) by intraperitoneally (i.p.) injection together with 45 minutes ischemia and 24 hours reperfusion). The period of Se administration was 2 weeks before the initiation of renal IR.

\section{Surgical procedure}

After the treatment period (2 weeks) with Se, the rats received ketamine $(75 \mathrm{mg} / \mathrm{kg})$ and xylazine $(8 \mathrm{mg} / \mathrm{kg})$ intraperitoneally (i.p.) to anesthetize. After that, the rats were prepared for the operation with shaving and disinfecting the abdominal area. Then, the surgical procedure was initiated by the development of an abdominal incision. To develop ischemia, the left and right kidney arteries were obstructed by using clamps for 45 minutes. After 45 minutes, the vessels were opened again and the renal blood flow was re-established. The reperfusion step was continued for 24 hours. Then, blood samples were prepared from the animal hearts and their serums were taken. Additionally, the removal of the left kidney was done immediately and homogenized for further biochemical analysis.

\section{The determination of renal functional parameters Urea and creatinine}

The measurement of renal functional parameters including urea and creatinine $(\mathrm{Cr})$ in the serum samples were performed by a biochemical autoanalyzer (Olympus AU-600, Tokyo, Japan). Commercial kits were used in this measurement.

\section{Lipid peroxidation measurement \\ Malondialdehyde}

Malondialdehyde (MDA), as lipid peroxidation (LPO) index, was measured by the thiobarbituric acid (TBA) method. The absorbance measurement was done spectrophotometrically at $540 \mathrm{~nm}$ wavelength. MDA concentration was shown as nM MDA/mg-pr (7).

The evaluation of oxidative stress and inflammation biomarkers

Glutathione

Serum and renal GSH levels were measured by spectrophotometer (Tokyo, Japan) at $412 \mathrm{~nm}$ wavelength, based on the Ellman method (8). The GSH levels were indicated as $\mathrm{nM} / \mathrm{mg}$-pr.

Catalase

Catalase (CAT) activity in serum and kidney was measured by the Sinha method (9). The reaction began by adding up sample $(20 \mu \mathrm{L})$ in $30 \mathrm{mM}$ hydrogen peroxide $(2 \mathrm{~mL})$ in 50 $\mathrm{mM}$ potassium phosphate buffer ( $\mathrm{pH}$ 7.0). Enzyme units are shown as mmol/L of utilized $\mathrm{H} 2 \mathrm{O} 2$ per min $\mathrm{g}$ or $\mathrm{mL}$.

\section{Glutathione peroxidase}

Serum and renal GPx activities were determined in accordance with our previous study (10).

\section{Myeloperoxidase measurement}

Serum myeloperoxidase (MPO) activities were determined in accordance with our previous study (11).

\section{Nitric oxide measurement}

The measurement of nitric oxide (NO) level in the serum samples was performed by the evaluation of nitrite as the end product of NO. Nitrite evaluation was done based on the method of Giustarini et al (12).

Paraoxonase 1 measurement

Serum paraoxonase 1 (PON1) activities were determined 
in accordance with our previous study (13).

\section{Ethical issues}

All of the experimental protocols were conducted in accordance with the manuals of the Animal Ethics Committee of the Lorestan University of Medical Sciences. Additionally, all experimental protocols and steps of the tests were conducted in compliance with the regulations of the Research Ethics Committee of our university and Iranian Ethical Guidelines for the use of animals in research. Additionally all animal experiments were in accordance with protocols approved by the United States National Institutes of Health (NIH, 1978).

\section{Statistical analysis}

The results were expressed as mean \pm SD. The MannWhitney test was performed, as the basic test, by using the SPSS software version 21 to identify group differences. Significance was considered statistically at $P$ value $<0.05$.

\section{Results}

The effect of Se on the serum levels of $\mathrm{Cr}$ and urea in RIR rats

The level of $\mathrm{Cr}$ in serum is demonstrated in Figure 1. The level of serum Cr significantly increased in untreated IR rats compared with the control group ( $1.31 \pm 0.121$ versus $1.25 \pm 0.12 \mathrm{mg} / \mathrm{dL}, P=0.01)$. Se could significantly inhibit the increase of serum Cr level in the treatment group compared with the untreated IR group ( $1.15 \pm 0.10$ versus $1.31 \pm 0.121 \mathrm{mg} / \mathrm{dL} ; P=0.02$ ).

The level of urea in serum is demonstrated in Figure 2. The level of serum urea significantly increased in the untreated IR group compared with the control group (101.25 \pm 6.14 versus $87.3 \pm 7.21 \mathrm{mg} / \mathrm{dL} ; P=0.01)$. The level of serum urea in the treated group was significantly less than that of the untreated IR group (91.7 \pm 8.68 versus $101.25 \pm 6.14$ $\mathrm{mg} / \mathrm{dL} ; P=0.04)$.

\section{The effect of Se on serum and renal MDA levels in RIR} rats

The level of MDA in serum is indicated in Table 1.

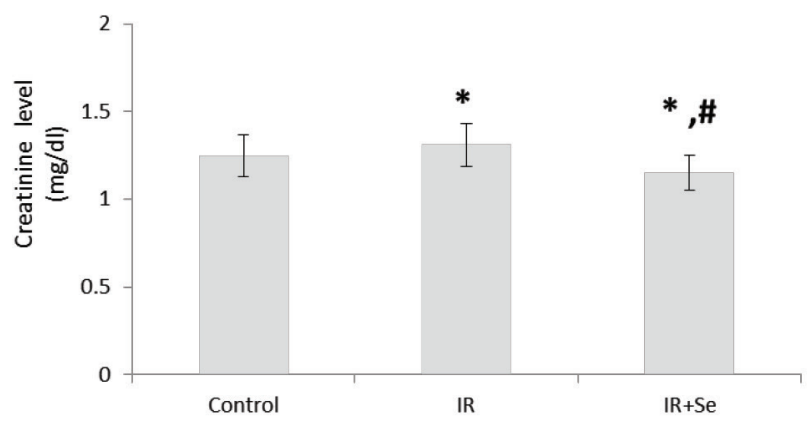

Figure 1. The effect of selenium (Se) on serum creatinine $(\mathrm{Cr})$ level in control, untreated and treated renal ischemia-reperfusion animals (RIR). Values are expressed as mean \pm standard deviation (SD). ${ }^{*} P<$ 0.05 compared with control group. \# $P<0.05$ compared with IR group.

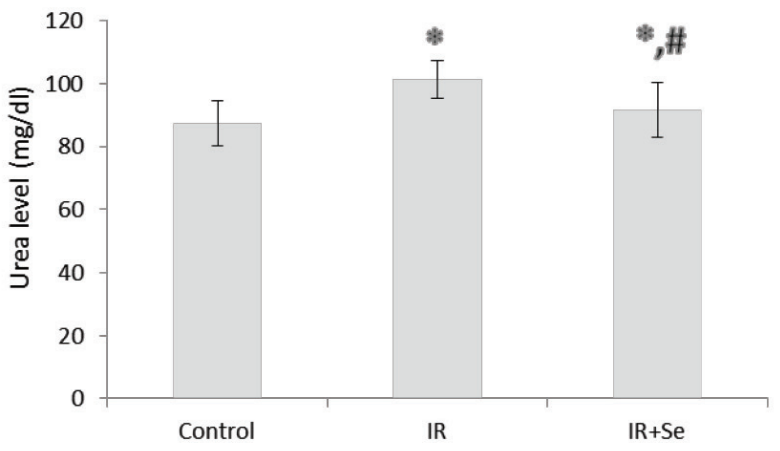

Figure 2. The effect of selenium (Se) on serum urea level in control, untreated and treated renal ischemia-reperfusion animals. Values are expressed as mean \pm standard deviation (SD). ${ }^{*} P<0.05$ compared with control group. \#P<0.05 compared with IR group.

The level of serum MDA significantly enhanced in the untreated IR group compared with the control group (53 \pm 4.34 versus $23.5 \pm 2.18 \mathrm{nM} / \mathrm{mg}$-pr; $P=0.01)$. The level of the serum MDA significantly reduced in the treated group compared with the untreated IR group $(35 \pm 2.97$ versus $53 \pm 4.34 \mathrm{nM} / \mathrm{mg}$-pr; $P=0.04)$.

The level of MDA in the kidney is indicated in Table 2. The level of renal MDA significantly increased in the untreated IR group compared with the control group (76.6 \pm 6.41 versus $36.0 \pm 3.38 \mathrm{nM} / \mathrm{mg}$-pr; $P=0.02$ ). The level of renal MDA significantly decreased in the treated group compared with the untreated IR group $(48.7 \pm 4.12$ versus $76.6 \pm 6.41 \mathrm{nM} / \mathrm{mg}$-pr; $P=0.03)$.

The effect of Se on serum and renal GSH levels in RIR rats

The level of GSH in serum is demonstrated in Table 1. The level of serum GSH significantly decreased in the untreated IR group compared with the control group (5.41 \pm 0.45 versus $11.3 \pm 1.0 \mathrm{nM} / \mathrm{mg}$-pr; $P=0.02$ ). The level of serum GSH significantly increased in the treated group compared with the untreated IR group $(9.2 \pm 0.86$ versus $5.41 \pm 0.45 \mathrm{nM} / \mathrm{mg}$-pr; $P=0.04$ ).

The level of GSH in the kidney is demonstrated in Table 2. The level of kidney GSH significantly reduced in the untreated IR group compared with the control group (6.2 \pm 0.59 versus $10.7 \pm 1.5 \mathrm{nM} / \mathrm{mg}$-pr; $P=0.01)$. Se could significantly increase the renal GSH level in the treated group in comparison to the untreated IR group $(9.7 \pm 0.87$ versus $6.2 \pm 0.59 \mathrm{nM} / \mathrm{mg}$-pr; $P=0.03$ ).

\section{The effect of Se on serum and renal CAT activities in RIR rats}

Serum CAT activity is indicated in Table 1. Serum CAT activity in the IR group was significantly lower than that of the control group $(58.1 \pm 4.61$ versus $140.5 \pm 13.84$ $\mathrm{U} / \mathrm{mg}$ protein; $P=0.03$ ). Se could significantly increase serum CAT activity in the treated group compared to the untreated IR group $(126.8 \pm 11.84$ versus $58.1 \pm 4.61 \mathrm{U} /$ 
Table 1. The effects of selenium administration on serum levels of MDA, GSH, serum activities of CAT, GPX and PON1 in control, un-treated and treated RIR rats

\begin{tabular}{llll}
\hline MDA (nmol/mg protein) & $2.18 \pm 23.5$ & $4.34 \pm 53^{*}$ & $2.97 \pm 35^{*, \#}$ \\
GSH (nmol/mg protein) & $1.0 \pm 11.3$ & $0.45 \pm 5.41^{*}$ & $0.86 \pm 9.2^{*, \#}$ \\
CAT (U/mg protein) & $13.84 \pm 140.5$ & $4.61 \pm 58.1^{*}$ & $11.84 \pm 126.8^{*, \#}$ \\
GPX (U/mg protein) & $41.22 \pm 428.83$ & $23.73 \pm 251.6^{*}$ & $40.22 \pm 420.3^{*, \#}$ \\
PON1 (U/min) & $40.34 \pm 19.51$ & $10.37 \pm 3.38^{*}$ & $19.92 \pm 14.91^{*}$ \\
\hline
\end{tabular}

Abbreviations: GPx, glutathione peroxidase; PON1, paraoxonase 1; RIR, renal ischemia-reperfusion; MDA, malondialdehyde; GSH, glutathione; CAT, catalase.

Values are expressed as mean \pm standard deviation (SD). ${ }^{*} P<0.05$ compared with control group. $\#<0.05$ compared with IR group.

Table 2. The effects of selenium administration on serum levels of MDA, GSH, serum activities of CAT, GPX and PON1 in cthe kidney of control, un-treated and treated RIR rats

\begin{tabular}{llll}
\hline Parameter & Control & IR & IR+Se \\
\hline MDA (nmol/mg protein) & $3.38 \pm 36$ & $6.41 \pm 76.6^{*}$ & $4.12 \pm 48.7^{*, \#}$ \\
GSH (nmol/mg protein) & $1.5 \pm 10.7$ & $0.59 \pm 6.2^{*}$ & $0.87 \pm 9.7^{*, \#}$ \\
CAT (U/mg protein) & $13.82 \pm 148.1$ & $9.97 \pm 113.4^{*}$ & $12.69 \pm 147.6^{*}, \#$ \\
GPX (U/mg protein) & $57.23 \pm 596.3$ & $40.2 \pm 428.7^{*}$ & $51.42 \pm 528.1^{*, \#}$ \\
\hline
\end{tabular}

Abbreviations: GPx, glutathione peroxidase; PON1, paraoxonase 1; RIR, renal ischemia-reperfusion; Se, Selenium; MDA, malondialdehyde; GSH, glutathione; CAT, catalase.

Values are expressed as mean \pm standard deviation (SD). ${ }^{*} P<0.05$ compared with control group. ${ }^{\#} P<0.05$ compared with IR group.

mg protein; $P=0.04)$. Serum CAT activity in the IR group treated with Se was similar to the control group.

Renal CAT activity is demonstrated in Table 2. Renal CAT activity significantly reduced in the untreated IR group compared with the control group (113.4 \pm 9.97 versus $148.1 \pm 13.82 \mathrm{U} / \mathrm{mg}$ protein; $P=0.03)$. Renal CAT activity in the IR group treated with Se was significantly more than that of the IR group $(147.6 \pm 12.694$ versus $113.4 \pm$ $9.97 \mathrm{U} / \mathrm{mg}$ protein; $P=0.03$ ). It was similar to renal CAT activity in the control group.

The effect of Se on serum and renal activities of GPX in RIR rats

Serum and renal GPX activities are respectively demonstrated in Tables 1 and 2. The serum GPX activity in the IR group was significantly lower than that of the control group $(251.6 \pm 32.73$ versus $428.83 \pm 41.22 \mathrm{U} /$ mg protein; $P=0.03$ ). Se could significantly enhance the serum GPX activity in the treated IR group compared with the untreated IR group $(420.3 \pm 40.22$ versus 251.6 $\pm 23.73 \mathrm{U} / \mathrm{mg}$ protein; $P=0.04$ ). The GPX activity in the serum of the IR group treated with Se was approximately similar to the GPX activity in the control group.

Renal GPX activity in the IR group was significantly lower than that of the control group (428.7 \pm 40.2 versus 596.3 $\pm 57.23 \mathrm{U} / \mathrm{mg}$ protein; $P=0.02$ ). Se could significantly increase renal GPX activity in the treated group in comparison to the untreated IR group $(528.1 \pm 51.42$ versus $428.7 \pm 40.2 \mathrm{U} / \mathrm{mg}$ protein; $P=0.01$ ). Renal GPX activity in the IR group treated with Se was approximately similar to the control group.

The effect of Se on the serum activity of PON 1 in RIR rats The serum PON1 activity is demonstrated in Table 1 . The serum PON1 activity in the IR group was significantly lower than that of the control group $(10.37 \pm 3.38$ versus $40.34 \pm 19.51 \mathrm{U} / \mathrm{mg}$ protein; $P=0.001)$. The PON1 activity in the serum of the treated group was more than that of the untreated IR group $(19.92 \pm 14.91$ versus $10.37 \pm$ $3.38 \mathrm{U} / \mathrm{mg}$ protein; $P=0.23$ ), but it was not statistically significant.

The effect of Se on the serum activity of MPO in RIR rats The level of MPO in the serum is demonstrated in Figure 3. The serum MPO activity in the IR group was significantly more than that of the control group (89.57 \pm 17.88 versus $56.75 \pm 18.87 \mathrm{U} / \mathrm{mg}$ protein; $P=0.017)$. Se could reduce the MPO activity in the serum of the treated group compared with the untreated IR group (74.20 \pm 29.02 versus $89.57 \pm 17.88 \mathrm{U} / \mathrm{mg}$ protein; $P=0.2$ ), but this change was not statistically significant.

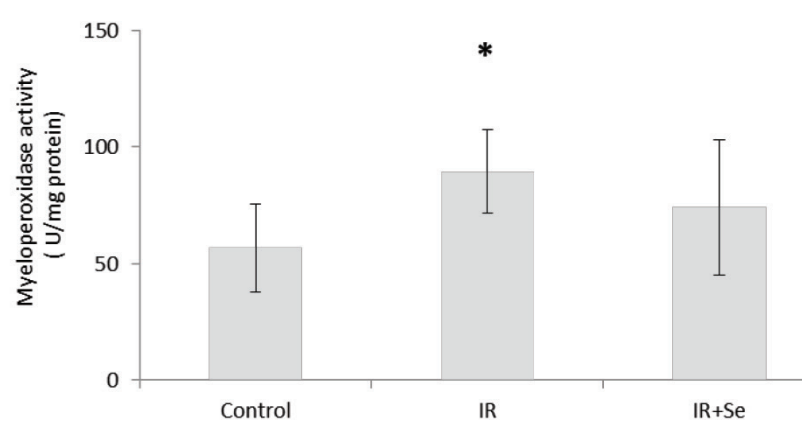

Figure 3. The effect of selenium (Se) on serum myeloperoxidase (MPO) activity in control, untreated and treated renal ischemia-reperfusion (RIR) animals. Values are expressed as mean \pm standard deviation (SD). ${ }^{*} P<0.05$ compared with control group. ${ }^{\sharp} P<0.05$ compared with IR group. 
The effect of Se on the serum NO level in RIR rats

The level of NO in the serum is demonstrated in Figure 4. The level of serum NO significantly increased in the untreated IR group compared with the control group (3.00 \pm 0.96 versus $1.80 \pm 0.52 \mathrm{nmol} / \mathrm{dL} ; P=0.007)$. Se could significantly decrease the level of serum NO in the treated group compared with the untreated IR group (1.95 \pm 0.39 versus $3.00 \pm 0.96 \mathrm{nmol} / \mathrm{dL} ; P=0.015)$. The serum level of NO in the treatment group was similar to the control group.

\section{Discussion}

RIRI is the major reason for AKI. Oxidative stress and inflammation are factors that create RIRI. Among these factors, oxidative stress particularly ROS plays a more important role than other factors in renal IR pathogenesis (1-3). Hence, the administration of antioxidants is considered as a therapeutic solution in order to protect organs against free radicals.

\section{The effect of Se on serum urea and $\mathrm{Cr}$}

Our results indicated that the levels of serum $\mathrm{Cr}$ and urea significantly increased in untreated IR rats compared with control rats. Se could significantly inhibit the increase of serum $\mathrm{Cr}$ and urea levels in the treatment group compared with the untreated IR group. A related study showed that curcumin could play an effective role in the reduction of renal IR damage via the significant decrease of urea level (14). Garlic oil was another treatment that its application significantly decreased $\mathrm{Cr}$ and urea levels (15). Aqueous garlic extract was also used for RIRI treatment and has similar effects on $\mathrm{Cr}$ and urea levels (16). It can be guessed that the reduction of renal function parameters in the serum may be due to antioxidant properties of Se because other researches demonstrated that natural antioxidants such as $\alpha$-tocopherol (17) and oxytocin (18) showed similar effects on $\mathrm{Cr}$ and urea levels. Hence, the administration of antioxidants such as Se with protective effects on renal functional parameters can ameliorate renal IR which induces renal functional damage.

\section{The effect of Se on serum and renal LPO}

Other results of our study showed that the levels of serum and renal MDA significantly enhanced in the untreated IR group compared with the control group. The levels of the serum and renal MDA significantly reduced in the treated group compared with the untreated IR group. MDA was measured as an LPO marker in this study. Various treatments were used in different studies that improved the increase of MDA level. Some of them are melatonin (19), oxytocin (18), curcumin (14), aqueous garlic extract (16). Previous studies revealed that Se could inhibit LPO in vivo (7). Our treatment could reduce significantly the serum and renal MDA levels similar to previous studies. Therefore, the use of natural antioxidants with beneficial effects on MDA can prevent or be helpful in decreasing

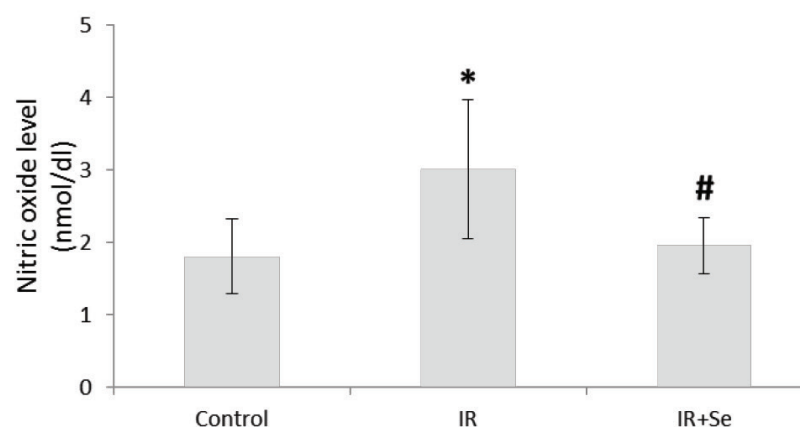

Figure 4. The effect of selenium (Se) on serum nitric oxide (NO) level in control, untreated and treated renal ischemia-reperfusion (RIR) animals. Values are expressed as mean \pm standard deviation (SD). ${ }^{*} P<0.05$ compared with control group. ${ }^{\#} P<0.05$ compared with IR group

complications that are associated with oxidative stress in RIRI.

The effect of Se on antioxidant enzyme activities and GSH level

The activities of GPX and CAT in serum and kidney, serum PON1 activity, serum and renal GSH levels significantly decreased in the untreated IR group compared with the control group. The treatment of animals with Se could increase the activities of GPX and CAT in serum and kidney, serum and renal GSH levels in the treated group compared with the untreated IR group. Serum PON1 activity also increased in the treated group, but it was not significant. The activities of CAT, GPX are used for the evaluation of antioxidant status. PON1 is an antioxidant enzyme that inhibits oxidative modification of LDL (Ox-LDL) and plays the main role in most of antioxidative activity that has been attributed to HDL (20). In various studied, other treatments including sitagliptin (21), curcumin (14), silymarin (22), and coenzyme Q10 (23) could exert protective effects on antioxidant defense system. A recent study also indicated that Se could improve the activities of antioxidant enzymes in vivo (24). Our results are similar to other researches which showed the beneficial effects of natural antioxidants on the antioxidant defense system. Hence, the utilization of natural antioxidants which can increase the activities of antioxidant enzymes and GSH level can inhibit or ameliorate the complications of renal IR which induces oxidative stress.

\section{The effect of Se on serum NO level and MPO activity}

The serum MPO activity in the untreated group was significantly more than that of the control group. Se could reduce the MPO activity in the serum of the treated group compared with the untreated IR group, but it was not statistically significant. Se could decrease MPO activity in the treatment group in a way that its activity was not significantly different from MPO activity in the control group $(P>0.05)$. MPO is a heme protein that is 
released by neutrophils. It plays a role in inflammation and oxidative stress processes. MPO is recognized as a marker of neutrophil infiltration and accumulation (25). Several reports have been suggested various antioxidant treatments for improving the MPO activity $(16,18,21)$. A previous study showed that Se could reduce MPO activity in vivo (26). Other part of our study showed Se could significantly decrease the level of serum NO in the treated group compared with the untreated IR group. NO reacts with superoxide radicals. Peroxynitrite, a cellular oxidant, is produced in this reaction (27). Antioxidants including the combination of Se and vitamin E (28), curcumin (14), and sitagliptin (21) could improve NO level in pathological conditions. It has been demonstrated that Se could ameliorate NO level in vivo (24). Our results are similar to other researches which indicated the beneficial effect of natural antioxidants on MPO activity and NO level. Thus the use of antioxidants such as Se can ameliorate the complications of renal IR which are associated with oxidative stress and inflammation.

In summary, our study and other researches showed that natural antioxidants have beneficial effects on antioxidant and inflammatory indices in renal IR injuries. On the other hand, natural antioxidants do not have side effects. Thus, we suggest that Se, as natural antioxidant, with protective effects on renal IR injuries must be considered as a helpful therapeutic strategy for RIR complications.

\section{Conclusion}

Se could improve renal function markers, LPO, the activities of antioxidant enzymes, GSH, NO, and MPO activity in renal IR rats. Therefore, Se, as a good antioxidant, can ameliorate renal IR complications which are associated with oxidative stress and inflammation.

\section{Acknowledgments}

This study was approved by the Lorestan University of Medical Sciences. The authors would like to give special thanks to the Lorestan University of Medical Sciences, Khorramabad, Iran, for their financial support. The authors also thank the head and staff of the Razi Herbal Medicines Research Center of Lorestan Medical University.

\section{Authors' contribution}

HA designed the project. EB, HN and ZZN; collected the data. HA analyzed the data. EB wrote the manuscript. HA revised the English version and edited the final draft.

\section{Conflicts of interest}

The authors declare that they have no conflict of interest.

Ethical considerations

Ethical issues (including plagiarism, data fabrication, double publication) have been completely observed by the authors.

\section{Funding/Support}

This research was supported by Lorestan University of Medical Sciences.

\section{References}

1. Kalogeris T, Baines CP, Krenz M, Korthuis RJ. Cell biology of ischemia/reperfusion injury. Int Rev Cell Mol Biol. 2012;298:229-317. doi: 10.1016/B978-0-12-3943095.00006-7.

2. Sharfuddin AA, Molitoris BA. Pathophysiology of ischemic acute kidney injury. Nat Rev Nephrol. 2011;7:189-200. doi:10.1038/nrneph.2011.16.

3. Granger DN, Kvietys PR. Reperfusion injury and reactive oxygen species: the evolution of a concept. Redox Biol. 2015;6:524-51. doi: 10.1016/j.redox.2015.08.020.

4. Hosnedlova B, Kepinska M, Skalickova S, Fernandez C, Ruttkay-Nedecky B, Malevu TD, et al. A summary of new findings on the biological effects of selenium in selected animal species-a critical review. Int J Mol Sci. 2017;18:2209. doi: 10.3390/ijms18102209.

5. Wang N, Tan HY, Li S, Xu Y, Guo W, Feng Y. Supplementation of micronutrient selenium in metabolic diseases: its role as an antioxidant. Oxid Med Cell Longev. 2017;2017:7478523. doi: $10.1155 / 2017 / 7478523$.

6. Iglesias P, Selgas R, Romero S, Díez JJ. Selenium and kidney disease. J Nephrol. 2013;26:266-72. doi: 10.5301/ jn. 5000213 .

7. Pourkhodadad S, Alirezaei M, Moghaddasi M, Ahmadvand H, Karami M, Delfan B, et al. Neuroprotective effects of oleuropein against cognitive dysfunction induced by colchicine in hippocampal CA1 area in rats. J Physiol Sci. 2016;66:397-405. doi: 10.1007/s12576-016-0437-4.

8. Ellman GL. Tissue sulfhydryl groups. Arch Biochem Biophys. 1959;82:70-7. doi: 10.1016/0003-9861(59)900906.

9. Sinha AK. Colorimetric assay of catalase. Anal Biochem. 1972;47:389-94. doi: 10.1016/0003-2697(72)90132-7.

10. Ahmadvand H, Ghasemi Dehnoo M, Cheraghi R, Rasoulian B, Ezatpour B, Azadpour M, et al. Amelioration of altered serum, liver, and kidney antioxidant enzymes activities by sodium selenite in alloxan-induced diabetic rats. Rep Biochem Mol Biol. 2014;3:14-20.

11. Khalatbary AR, Ahmadvand H. Effect of oleuropein on tissue myeloperoxidase activity in experimental spinal cord trauma. Iran Biomed J. 2011;15:164-7. doi: 10.6091/ IBJ.1026.2012.

12. Giustarini D, Rossi R, Milzani A, Dalle-Donne I. Nitrite and nitrate measurement by Griess reagent in human plasma: evaluation of interferences and standardization. Methods Enzymol. 2008;440:361-80. doi: 10.1016/S00766879(07)00823-3.

13. Ahmadvand H, Bagheri S, Tamjidi-Poor A, Cheraghi M, Azadpour M, Ezatpour B, et al. Effects of oleuropein on lipid peroxidation, lipid profile, atherogenic indices, and paraoxonase 1 status in gentamicin-induced nephrotoxicity in rats. ARYA Atheroscler. 2016;12:87-93.

14. Bayrak O, Uz E, Bayrak R, Turgut F, Atmaca AF, Sahin S, et al. Curcumin protects against ischemia/reperfusion injury in rat kidneys. World J Urol. 2008;26:285-91. doi: 10.1007/ s00345-008-0253-4.

15. Savas M, Yeni E, Ciftci H, Yildiz F, Gulum M, Keser BS, et al. The antioxidant role of oral administration of garlic oil on 
renal ischemia-reperfusion injury. Ren Fail. 2010;32:362-7. doi: $10.3109 / 08860221003611711$.

16. Kabasakal L, Şehirli Ö, Çetinel Ş, Cikler E, Gedik N, Şener G. Protective effect of aqueous garlic extract against renal ischemia/reperfusion injury in rats. J Med Food. 2005;8:319-26. doi: 10.1089/jmf.2005.8.319.

17. Rhoden EL, Pereira-Lima L, Telöken C, Lucas ML, BellóKlein A, Rhoden CR. Beneficial effect of a-tocopherol in renal ischemia-reperfusion in rats. Jpn J Pharmacol. 2001;87:164-6. doi: 10.1254/jjp.87.164.

18. Tuğtepe H, Șener G, Biyikli NK, Yüksel M, Çetinel Ş, Gedik $\mathrm{N}$, et al. The protective effect of oxytocin on renal ischemia/ reperfusion injury in rats. Regul Pept. 2007;140:101-8. doi: 10.1016/j.regpep.2006.11.026.

19. Sahna E, Parlakpinar H, Ozturk F, Cigremis Y, Acet A. The protective effects of physiological and pharmacological concentrations of melatonin on renal ischemia-reperfusion injury in rats. Urol Res. 2003;31:188-93. doi: 10.1007/ s00240-003-0314-5.

20. Aviram M, Kaplan M, Rosenblat M, Fuhrman B. Dietary antioxidants and paraoxonases against LDL oxidation and atherosclerosis development. Handb Exp Pharmacol. 2005; 170:263-300.

21. Vaghasiya J, Sheth N, Bhalodia Y, Manek R. Sitagliptin protects renal ischemia reperfusion induced renal damage in diabetes. Regul Pept. 2011;166:48-54. doi: 10.1016/j. regpep.2010.08.007.

22. Turgut F, Bayrak O, Catal F, Bayrak R, Atmaca AF, Koc A, et al. Antioxidant and protective effects of silymarin on ischemia and reperfusion injury in the kidney tissues of rats. Int Urol Nephrol. 2008;40:453-60. doi: 10.1007/ s11255-008-9365-4.

23. Ahmadvand H, Ghasemi Dehnoo M, Dehghani A, Bagheri S, Cheraghi RA. Serum paraoxonase 1 status and its association with atherogenic indexes in gentamicin-induced nephrotoxicity in rats treated with coenzyme Q10. Ren Fail. 2014;36:413-8. doi: 10.3109/0886022X.2013.865154.

24. Zendedel A, Delavari S, Ahmadvand H, Ghanadi K, Gholami M. Effects of selenium on antioxidant activity and recovery from sciatic nerve ischemia-reperfusion in adult rats. Zahedan J Res Med Sci. 2015;17:e5200. doi: 10.17795/ zjrms-5200.

25. Anatoliotakis N, Deftereos S, Bouras G, Giannopoulos G, Tsounis D, Angelidis C, et al. Myeloperoxidase: expressing inflammation and oxidative stress in cardiovascular disease. Curr Top Med Chem. 2013;13:115-38. doi: 10.2174/1568026611313020004.

26. Zolali E, Hamishehkar H, Maleki-Dizaji N, Majidi Zolbanin N, Ghavimi H, Kouhsoltani M, et al. Selenium effect on oxidative stress factors in septic rats. Adv Pharm Bull. 2014;4:289-93. doi: 10.5681/apb.2014.042.

27. Yagmurca $M$, Erdogan $H$, Iraz $M$, Songur A, Ucar M, Fadillioglu E. Caffeic acid phenethyl ester as a protective agent against doxorubicin nephrotoxicity in rats. Clin Chim Acta. 2004;348:27-34. doi: 10.1016/j.cccn.2004.03.035.

28. Aly HF, Mantawy MM. Comparative effects of zinc, selenium and vitamin $\mathrm{E}$ or their combination on carbohydrate metabolizing enzymes and oxidative stress in streptozotocine-induced diabetic rats. Eur Rev Med Pharmacol Sci. 2012;16:66-78.

Copyright (c) 2019 The Author(s); Published by Nickan Research Institute. This is an open-access article distributed under the terms of the Creative Commons Attribution License (http://creativecommons.org/licenses/by/4.0), which permits unrestricted use, distribution, and reproduction in any medium, provided the original work is properly cited. 